\title{
Design, optimization, and validation of chemometrics-assisted spectrophotometric methods for simultaneous determination of etodolac and thiocolchicoside in pharmaceuticals
}

\author{
Mevlut Albayrak $^{1 *}$ D, Fatma Demirkaya-Miloglu², Onur Senol ${ }^{2}$ and Elmas Polatdemir ${ }^{2}$
}

\begin{abstract}
Etodolac (ET) is a nonsteroidal anti-inflammatory drug (NSAID) that shows anti-inflammatory, analgesic, and antipyretic activities. Various NSAIDs have been demonstrated to enhance analgesia such as thiocolchicoside (TC) which is also an anti-inflammatory analgesic with muscle relaxant properties. A new quantitative method by using the data obtained from chemometrics calibration partial least squares (PLS), principal component regression (PCR), and inverse least squares (ILS) was developed for the simultaneous analysis of ET and TC without any separation process in combined pharmaceutical products. The calibration (25 samples) and validation (8 samples) concentration data matrices were prepared by using the mixtures containing ET $(15-75 \mu \mathrm{g} / \mathrm{mL})$ and TC $(1-10 \mu \mathrm{g} / \mathrm{mL})$ via factorial design method. The absorbance values were measured in the $240-440 \mathrm{~nm}$. The recovery $\%$ of PLS, PCR, and CLS methods were found as $\leq 98.26 \%, 98.16 \%$, and $98.17 \%$ for ET and $99.14 \%, 98.26 \%$, and $98.15 \%$ for TC, respectively. These three chemometric techniques, which do not require neither separation nor derivatization steps, were confidentially applied for the determination of ET and TC in combined formulations. In conclusion, it is claimed that these new chemometrics-assisted spectrophotometric methods are inexpensive, rapid, and simple and can be trustfully carried out in quality control laboratories as an alternative to existing methods.
\end{abstract}

Keywords: Chemometric calibration methods, Etodolac, Experimental design, Thiocolchicoside

\section{Introduction}

Etodolac (ET) which is a newly used nonsteroidal agent (NSAID) shows both anti-inflammatory and analgesic activity (Andelman 1983). It selectively inhibits cyclooxygenase-2 which is an important tool in the progression of inflammation (Hasegawa et al. 2005; Matsuyama et al. 2010; Riendeau et al. 1997) and is commonly used in diseases such as rheumatoid arthritis, osteoarthritis, and pain due to the minor surgery (Lynch and Brogden 1986). The second pharmaceutical that we have studied in our study is thiocolchicoside (TC), 2-demethoxy-2-glucosidoxythiocolchicine, which is a colchicine derivative. TC is one of the

\footnotetext{
* Correspondence: m_albayrak25@hotmail.com

1 Department of Medical Laboratory Techniques, Health Services Vocational

Training School, Ataturk University, 25240 Erzurum, Turkey

Full list of author information is available at the end of the article
}

myorelaxant drugs which has also anti-inflammatory and analgesic effect in spite of its major convulsant activity (Mascia et al. 2007). It has an antagonistic effect on GABA receptors which cause a convulsant activity (Biziere et al. 1981; Perucca et al. 1995; Sechi et al. 2003). These two drugs are used in combined form in order to provide a rapid analgesic effect on especially serious pain cases such as vertebral colon syndrome, severe trauma, and surgery operations. The combined form does not change the pharmacokinetic properties of both drugs. It has also a synergetic effect on the analgesic feature. So, it is very important to determine the concentration of each drug found in pharmaceutical preparations for quality control studies. There is no official method in any pharmacopeia for the simultaneous estimation of ET and TC (European Pharmacopoeia 6th edition 2007) via UV-Vis spectrophotometry. Literature survey reveals that several 
HPLC (Alagar et al. 2012; Dhiware et al. 2012; Patel and Shah 2014; Rathod and Patel 2012; Syamala 2016), HPTLC (Tekade et al. 2012), and spectrophotometric (Pandey et al. 2014; Thankappan et al. 2012; Tiwari and Pillai 2011) studies are available for the simultaneous analysis of ET and TC in pharmaceuticals. The spectrophotometric method is one of the most preferred approach for pharmaceutical analysis because it provides simplicity and inexpensiveness compared to other analytical methods due to natural native convenience and usefulness in most quality control studies of drugs. But conventional spectrophotometry method failed to perform the simultaneous analysis of several active substances in pharmaceuticals due to encountering in overlaps in a spectrum of multiple analytes which interfere each other and make it hard to predict their actual concentration. So, several different approach were developed to solve this problem like simultaneous equation (Pandey et al. 2014; Tiwari and Pillai 2011), Q-absorbance ratio (Pandey et al. 2014; Thankappan et al. 2012), and absorbance ratio (Tiwari and Pillai 2011) method which were used to resolve the overlapping spectrum of conventional spectrophotometry for the simultaneous determination of ET and TC. Recently, chemometrics techniques containing several multivariate calibration calculations (Riahi et al. 2010; Sankar et al. 2011) in combination with spectrophotometry have been applied to resolve the overlapping spectra of mixtures of drugs in pharmaceuticals because multivariate methods allow to filter interferences of analyzed substance and have several advantages such as lower usage of chemicals, manpower, less time consumed, reasonable accuracy, and precision without chromatographic separation procedures. To the best of our knowledge, there are no publications for the simultaneous determination of ET and TC based on the coupling of UV-Vis spectroscopy and multivariate calibration methods. This is the first study for the simultaneous analysis of ET and TC in combined pharmaceutical by using the chemometrics-assisted spectrophotometric methods based on the multivariate calibration techniques, mainly PLS, PCR, and ILS.

\section{Materials and methods}

A new quantitative method by using the data obtained from chemometrics calibration partial least squares (PLS), principal component regression (PCR), and inverse least squares (ILS) was developed for the simultaneous analysis of ET and TC without any separation process in combined pharmaceutical products. A Thermo Scientific Multiscan GO 51119300 model UV-Visible spectrophotometer was used for spectrophotometric measurements. The spectral bandwidth was settled at $1 \mathrm{~nm}$, and the wavelength scanning speed of the proposed method was $1000 \mathrm{~nm} / \mathrm{min}$. PLS, PCR, and ILS algorithms were conducted by using MATLAB R2014b (PLS-Toolbox software version 8.1.1). Design-Expert 8.0 (Stat-Ease Inc., Minneapolis, MN, USA) was selected to determine the calibration and validation set. Microsoft Excel 2010 and SPSS 11.5 software program (SPSS, Chicago, IL, USA) were used for the statistical analysis. Pharmaceutical grade of ET and TC was purchased from Sigma-Aldrich (Germany). Methanol used to prepare all solutions was HPLC grade (Merck, Germany). Etotio tablet (Mustafa Nevzat Pharmacy, Turkey) containing 400 $\mathrm{mg}$ of ET and $8 \mathrm{mg}$ of TC was selected for this study. Stock solutions of ET $(5 \mathrm{mg} / \mathrm{mL})$ and TC $(0.4 \mathrm{mg} / \mathrm{mL})$ were prepared by dissolving the compounds in methanol. The obtained solutions were protected against light and kept at $4{ }^{\circ} \mathrm{C}$. The working solutions were prepared by diluting the respective stock solutions of ET and TC. In order to find the linearity range for both $\mathrm{ET}$ and $\mathrm{TC}$, the stock solution was diluted with methanol and the absorption spectra of obtained solutions were recorded over the range of $240-440 \mathrm{~nm}$. ET and TC showed maximum absorbance at $280 \mathrm{~nm}$ and $378 \mathrm{~nm}$, respectively. In addition to this, $400 \mathrm{mg}$ of ET was combined with $8 \mathrm{mg}$ of TC according to the proposed fixed-dose combination. So, the linearity ranges were selected as $15-75 \mu \mathrm{g} / \mathrm{mL}$ for ET and $1-10 \mu \mathrm{g} / \mathrm{mL}$ for TC. Working solutions containing different concentrations of each compound were prepared in two sets. These were the calibration ( 25 samples) and validation (8 samples) sets which were determined by design expert 8.0 software under factorial design for 2 categories at 5 and 3 levels of each category, respectively. Absorption spectra of obtained sets were recorded between wavelengths of 240 to $440 \mathrm{~nm}$ with the intervals $\Delta \lambda=1$ nm.

\section{Pharmaceutical sample preparation}

Twenty different tablets, each containing $400 \mathrm{mg}$ of ET and $8 \mathrm{mg}$ of TC, were accurately weighed and grinded to form a powder. The weight of the obtained powder is just equal to one tablet which was dissolved in methanol and sonicated for $15 \mathrm{~min}$. The drug solution was filtered via a $0.5-\mu \mathrm{m}$ membrane filter and diluted for UV measurements.

\section{Results and discussion}

\section{Spectral analysis of ET and TC}

To determine the highest absorbance for ET and TC, several solvents containing water, ethanol, methanol, acetonitrile, and diethyl ether were tried, and finally, methanol was selected. Individual UV absorption spectra of ET and TC, together with a mixture of them, in the standard solutions at their nominal concentrations were recorded between 240 and $440 \mathrm{~nm}$ in methanol. It can be observed that ET and TC have overlapped spectra although wavelength shown the maximum absorbance of standard ET and TC as $278 \mathrm{~nm}$ and $370 \mathrm{~nm}$, respectively (Fig. 1a and b). The presence of overlapping spectra throughout the wavelength range prevents the analysis 


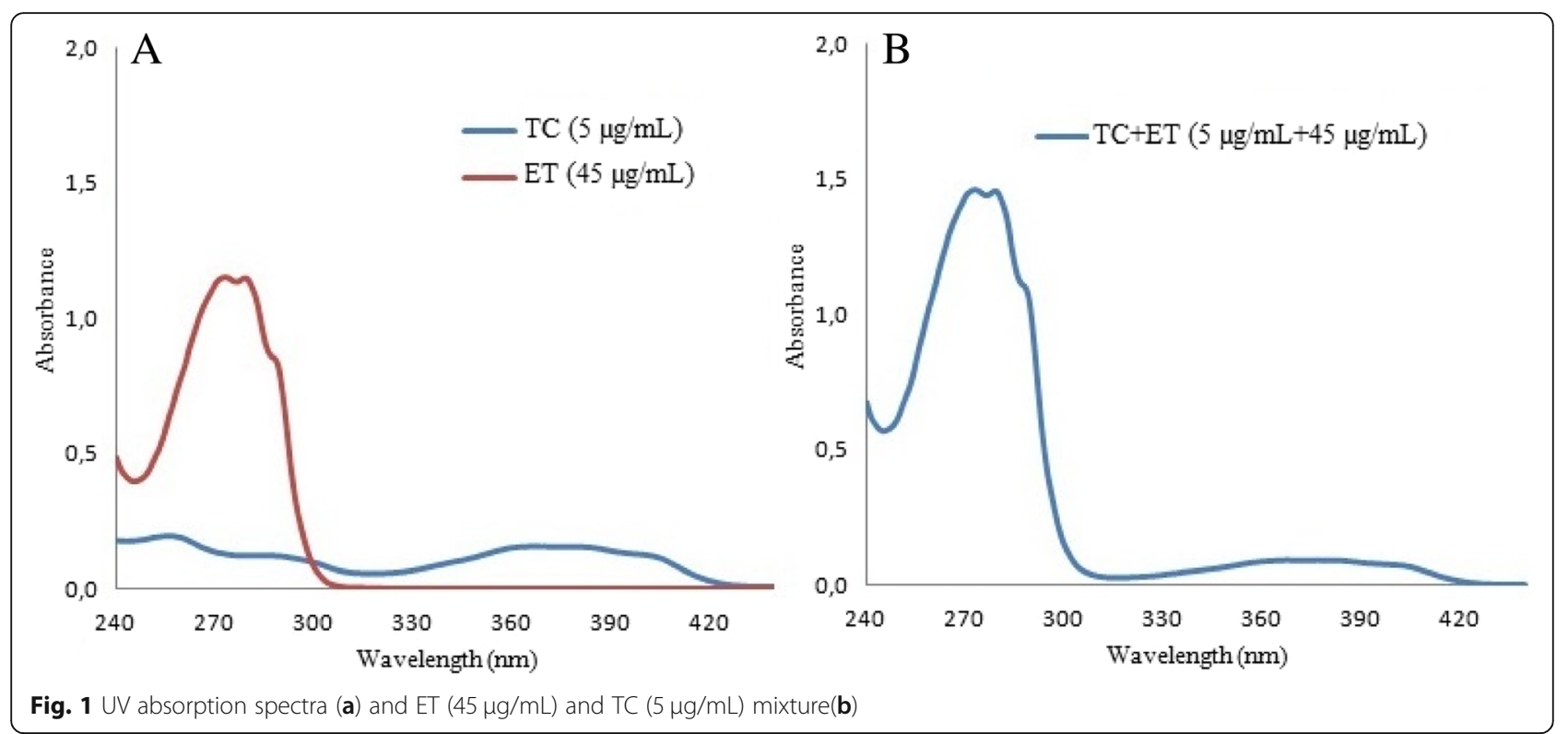

of the sample via traditional spectrophotometric methods which forced analysts to use a separation technique. Nowadays, spectrophotometric analyses of mixtures having overlapping absorption peaks were easily done by chemometrics-assisted calibration techniques. In this work, PLS, PCR, and ILS chemometric calibrations were established by using absorbance values measured at 200 points between 240 and $440 \mathrm{~nm}$ wavelength spectral region that provides sufficient information for the quantitative determination of ET and TC (with data points taken every $1 \mathrm{~nm}$ ).

\section{Construction of PLS, PCR, and ILS methods}

ILS is described as the inverse of Beer's Law where concentrations of an analyte are structured to be a function of absorbance measurements. The coefficient matrix $(P)$ was calculated from the linear equation regression by exporting the absorbance values and the calibration set in ILS method. Embedding $P$ matrix into the equation system gives the ILS calibrations. PLS is a soft modeling technique by recording the absorbance that explains the multidimensional data of dependent and independent variables in a two-dimensional diagram via latent variables (Dinç and Baleanu 2002). PCR is known as a combination of the principal component analysis (PCA) and ILS to form a quantitative model for complex samples. PLS is closely related to PCR. PCR methods reveal response variables from factors underlying the predictive variables of principal component analysis. However, the PLS algorithm selects $X$-scores of the latent independents which are matched as much as possible with $Y$-scores of the latent response variable(s) in order to get higher variance value, and PCR

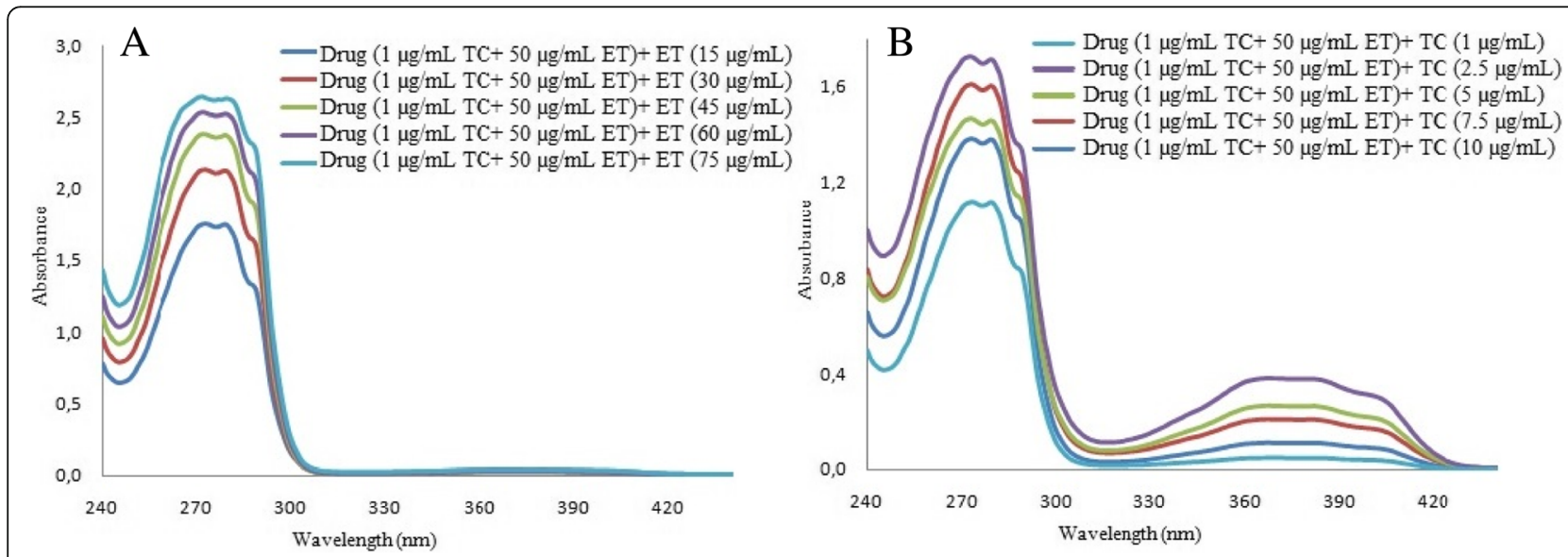

Fig. 2 UV absorption spectra of the standard solutions added to the pharmaceutical preparation for ET (a) and TC (b) 
Table 1 Obtained statistical values for the simultaneous analysis of ET and TC multivariate calibration methods (PLS, PCR, and ILS)

\begin{tabular}{|c|c|c|c|c|c|c|}
\hline Parameters & \multicolumn{3}{|c|}{ Thiocolchicoside } & \multicolumn{3}{|l|}{ Etodolac } \\
\hline Concentration range $(\mu \mathrm{g} / \mathrm{mL})$ & \multicolumn{3}{|l|}{$1-10$} & \multicolumn{3}{|l|}{$15-75$} \\
\hline Spectral region (nm) & \multicolumn{3}{|l|}{$240-440$} & \multicolumn{3}{|l|}{$240-440$} \\
\hline Cross-validation results & PLS & $P C R$ & ILS & PLS & PCR & ILS \\
\hline Optimum number of factors & 4 & 5 & - & 4 & 6 & - \\
\hline Slope & 1.0043 & 0.9925 & 1.025 & 0.9974 & 0.9880 & 0.9960 \\
\hline Intercept & -0.0237 & 0.0003 & 0.097 & 0.2963 & 0.5764 & 0.0007 \\
\hline$R^{2}$ & 0.9998 & 0.9997 & 0.9991 & 0.9999 & 0.9994 & 0.9998 \\
\hline RMSE-CV ( $\mu \mathrm{g} / \mathrm{mL})$ & 0.3686 & 0.4113 & - & 2.7289 & 3.6878 & - \\
\hline RMSEC & 0.2447 & 0.2620 & 0.7372 & 2.3330 & 2.9049 & 2.6381 \\
\hline RMSEP & 0.6092 & 0.3050 & - & 5.2988 & 2.8973 & - \\
\hline SEC & - & - & 0.6036 & - & - & 2.5262 \\
\hline \multicolumn{7}{|l|}{ Validation Results } \\
\hline Slope & 0.9956 & 1.0076 & 1.010 & 1.0019 & 1.0009 & 1.013 \\
\hline Intercept & 0.0640 & -0.0187 & 0.018 & 0.0027 & 0.1834 & 0.854 \\
\hline$R^{2}$ & 0.9993 & 0.9992 & 0.9995 & 0.9991 & 0.9993 & 0.9997 \\
\hline RMSEC & 0.2047 & 0.2445 & 0.6036 & 1.692 & 1.295 & 2.5262 \\
\hline
\end{tabular}

is based on predicting the maximum proportion of factor variation by observing $X$-scores (Sorouraddin et al. 2011). In this context, PLS, PCR, and ILS algorithms were applied with the absorbance and concentration matrix of 200 points in the range of $240-440$ $\mathrm{nm}$ for calibration set and validation sets by using PLS-toolbox 2.1 software. The cross-validation algorithm was carried out to discard only one analyte at a time, and then, the left standard spectra were monitored by PLS, ILS, and PCR. By the aid of this technique, the concentration of the rest sample could be successively predicted. This step was renewed till each of them had been left for once. For an accurate and precise prediction, a visual inspection could be an approach to determine the ideal number of a factor which is also known as latent variables. For PCR and

Table 2 Analysis results of ET and TC in the validation set using multivariate calibration methods (PLS, PCR, and ILS)

\begin{tabular}{|c|c|c|c|c|c|c|c|c|}
\hline \multirow{3}{*}{$\begin{array}{l}\text { Sample } \\
\text { no. }\end{array}$} & \multirow{2}{*}{\multicolumn{2}{|c|}{$\begin{array}{l}\text { Added } \\
\text { concentration } \\
(\mu \mathrm{g} / \mathrm{mL})\end{array}$}} & \multicolumn{6}{|c|}{ Found concentration $(\mu \mathrm{g} / \mathrm{mL})$} \\
\hline & & & \multicolumn{2}{|l|}{ PLS } & \multicolumn{2}{|l|}{$P C R$} & \multicolumn{2}{|l|}{ ILS } \\
\hline & $\overline{\mathrm{ET}}$ & $\mathrm{TC}$ & ET & $\mathrm{TC}$ & $\overline{\mathrm{ET}}$ & TC & $\overline{\mathrm{ET}}$ & $\mathrm{TC}$ \\
\hline 1 & 45 & 1.0 & 47.07 & 1.03 & 47.15 & 0.98 & 47.67 & 0.98 \\
\hline 2 & 60 & 2.5 & 59.05 & 2.63 & 58.95 & 2.52 & 58.37 & 2.47 \\
\hline 3 & 30 & 2.5 & 30.85 & 2.46 & 31.39 & 2.47 & 30.53 & 2.44 \\
\hline 4 & 75 & 5.0 & 75.88 & 5.15 & 75.72 & 4.98 & 77.98 & 5.15 \\
\hline 5 & 15 & 5.0 & 15.49 & 5.10 & 14.85 & 4.97 & 15.28 & 5.15 \\
\hline 6 & 60 & 7.5 & 61.25 & 7.52 & 59.80 & 7.85 & 60.83 & 7.87 \\
\hline 7 & 30 & 7.5 & 31.46 & 8.00 & 30.55 & 7.77 & 31.54 & 7.66 \\
\hline 8 & 45 & 10.0 & 44.81 & 9.80 & 44.22 & 9.97 & 46.98 & 9.67 \\
\hline
\end{tabular}

PLS algorithm, latent variables were selected to achieve better performance in prediction before constructing the models. Latent variables for both methods were found by calculating the prediction error sum of squares (PRESS $\left.=\Sigma\left(C_{\text {act }}-C_{\text {pred }}\right)^{2}\right)$. Bias was evaluated by the cross-validated models by using the optimum number of factors. The concentration of each active substances was calculated for each sample that was discussed by comparing actual concentrations in these calibration samples, and rootmean-square errors of cross-validation (RMSECV $=\sqrt{ }($ PRESS $/ n))$ were also measured for PCR and PLS models. In addition to these, the standard error of calibration and prediction (SEC $(\mathrm{SEP})=n \sum\left(C_{\text {act }}\right.$ $\left.-C_{\text {pred }}\right)^{2} n-1$ ) was calculated for predictions of the standard variation of each calibration in the drug mixtures and it was the correlation coefficient $\left(R^{2}\right)$ that was identified for each method, where $C_{\text {pred }}, C_{\text {act }}$, and $n$ are the predicted concentrations of drug samples, actual concentrations, and analysis number, respectively. Four latent variables were selected to be optimum to explain the model for both ET and TC via PLS, and six latent variables for ET and five latent variables for TC were the optimum for PCR method (Fig. 2). PLS, PCR, and ILS calibrations were obtained by plotting the added standards against the predicted amount by using the concentrations and absorbance data matrix. A satisfactory correlation coefficient $\left(R^{2}\right)$ value, low RMSECV, was calculated for PCR and PLS and SEC value for ILS indicating good predictive abilities of the models, and other statically parameters for cross-validation were obtained for each compound 


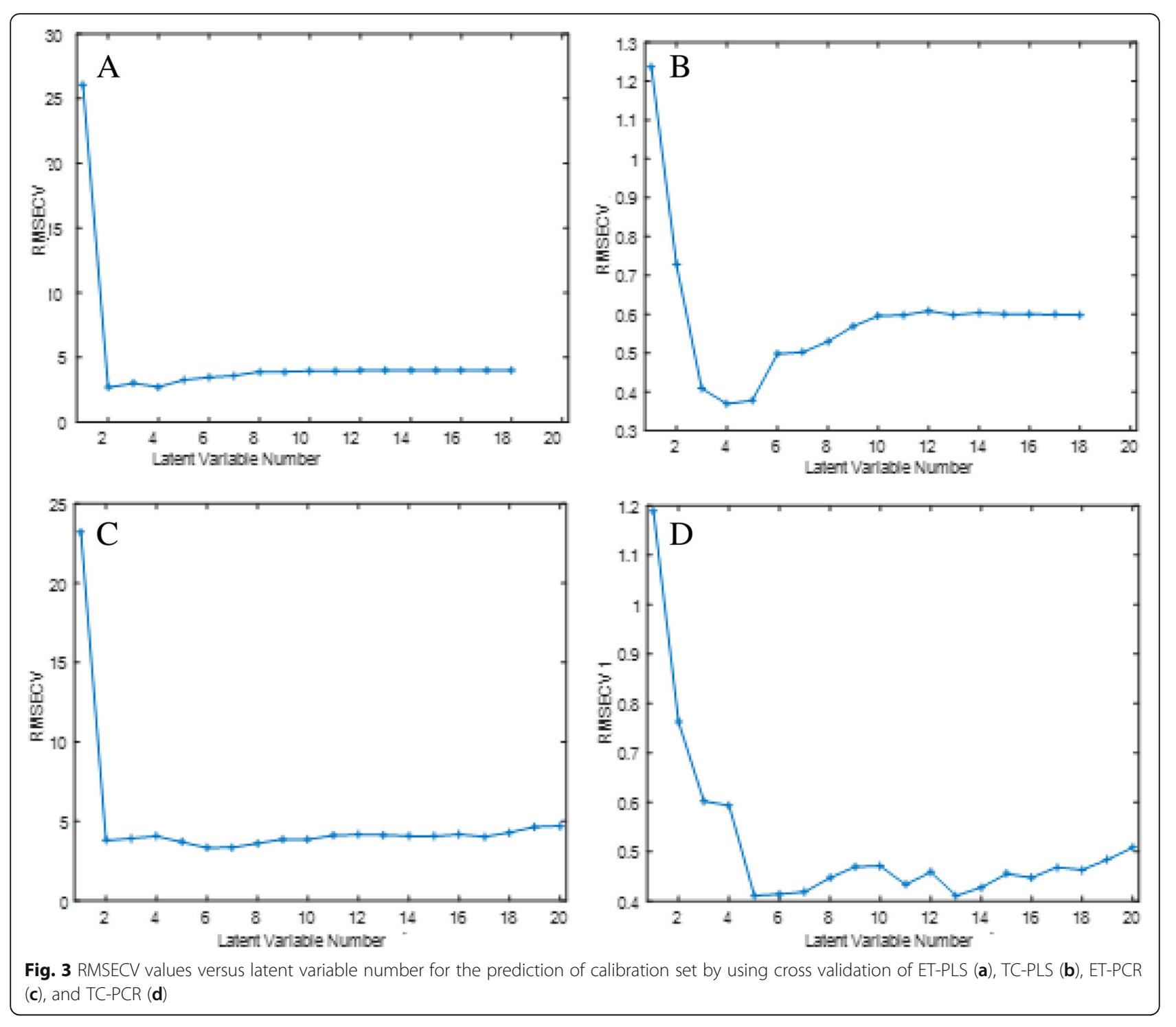

Table 3 Correlation matrix and $p$ values via Pearson for ET and TC

\begin{tabular}{|c|c|c|c|c|c|c|c|c|c|}
\hline \multirow[t]{2}{*}{ Variables } & & \multicolumn{4}{|l|}{ ET } & \multicolumn{4}{|l|}{ TC } \\
\hline & & Added & PLS & $P C R$ & ILS & Added & PLS & $P C R$ & ILS \\
\hline \multirow[t]{4}{*}{ Correlation matrix } & Added & 1 & 0.998 & 0.999 & 0.998 & 1 & 0.999 & 0.998 & 0.997 \\
\hline & PLS & 0.998 & 1 & 0.998 & 0.998 & 0.999 & 1 & 0.999 & 0.999 \\
\hline & PCR & 0.999 & 0.998 & 1 & 0.999 & 0.998 & 0.999 & 1 & 0.998 \\
\hline & ILS & 0.998 & 0.998 & 0.999 & 1 & 0.997 & 0.999 & 0.998 & 1 \\
\hline \multirow[t]{4}{*}{$p$ values } & Added & 0 & $<0.0001$ & $<0.0001$ & $<0.0001$ & 0 & $<0.0001$ & $<0.0001$ & $<0.0001$ \\
\hline & PLS & $<0.0001$ & 0 & $<0.0001$ & $<0.0001$ & $<0.0001$ & 0 & $<0.0001$ & $<0.0001$ \\
\hline & PCR & $<0.0001$ & $<0.0001$ & 0 & $<0.0001$ & $<0.0001$ & $<0.0001$ & 0 & $<0.0001$ \\
\hline & ILS & $<0.0001$ & $<0.0001$ & $<0.0001$ & 0 & $<0.0001$ & $<0.0001$ & $<0.0001$ & 0 \\
\hline
\end{tabular}


(Table 1). Furthermore, the validation sets which contained eight samples were analyzed by the mentioned procedure. All results about the prediction of those algorithms are summarized in Table 2. Similarly, the RMSEC value for validation set is reasonably low to each analyte indicating good accuracy and precision.

\section{Validation of the of PLS, PCR, and ILS methods}

For the validation of the multivariate calibration methods, the selectivity, sensitivity accuracy, and precision of each calibration method were established by using the validation set prepared from mixtures containing ET and TC that were tested. The validation set was analyzed with procedures described under the PLS, PCR, and ILS calibration methods. The obtained results were satisfactory (Table 2). The proposed calibration methods for the simultaneous determination of ET and TC had high selectivity. The results of the precision of the methods were given as the percent relative standard deviation (RSD \%), where results of the accuracy of methods were given as the relative error (RE). The RSD $\%$ values for the PLS, PCR, and ILS methods were found to be $2.64 \%, 3.01 \%$, and $3.29 \%$ for ET and $3.02 \%, 3.76 \%$, and $6.50 \%$ for TC, respectively. The RE values for the PLS, PCR, and ILS methods were determined to be 2.52, 0.29 , and 3.55 for ET and 2.09, 0.65, and 1.71 for TC, respectively. In addition to this, recoveries (R\%) were studied by spiking the known quantities of ET $(15,30,45$, 60 , and $75 \mu \mathrm{g} / \mathrm{mL})$ and $\mathrm{TC}(1.0,2.5,5.0,7.5$, and $10.0 \mu \mathrm{g} /$ $\mathrm{mL}$ ) with necessary dilutions in pharmaceutical tablet (Etotio ${ }^{\circ}$ containing $400 \mathrm{mg}$ of ET and $8 \mathrm{mg}$ of $\mathrm{TC}$ ) (Fig. 3). The R\% of PLS, PCR, and CLS methods were found as $\leq 98.26 \%, 98.16 \%$, and $98.17 \%$ for ET and 99.14\%, $98.26 \%$, and $98.15 \%$ for TC indicating good accuracy. In addition to this, the limit of detection (LOD $\left.\left.=3\left\|\delta_{\mathrm{r}}\right\|\right)\left\|b_{\mathrm{k}}\right\|\right)$ ) have been determined for the sensitivity of multivariate calibration methods, where $\|$.$\| is the Euclidean$ norm, $b_{\mathrm{k}}$ is the vector of regression coefficients determined by the calibration model, and $\left\|\delta_{\mathrm{r}}\right\|$ is the instrumental noise. The LOD values for PLS, PCR, and ILS method were $0.49 \mu \mathrm{g} / \mathrm{mL}, \quad 0.52 \mu \mathrm{g} / \mathrm{mL}$, and $0.58 \mu \mathrm{g} / \mathrm{mL}$ for ET and $0.22 \mu \mathrm{g} / \mathrm{mL}, \quad 0.28 \mu \mathrm{g} / \mathrm{mL}$, and $0.34 \mu \mathrm{g} / \mathrm{mL}$ for $\mathrm{TC}$, respectively.

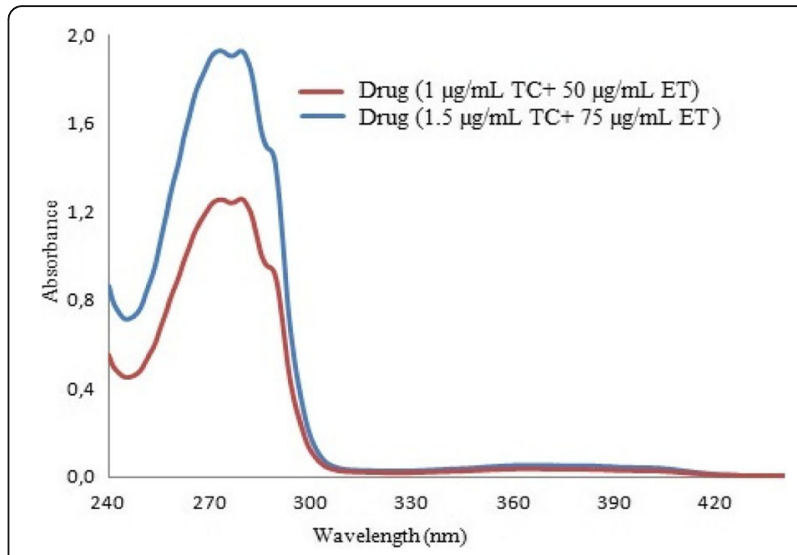

Fig. 4 UV absorption spectra of the drug (Etotio tablets) solutions containing ET $(50 \mu \mathrm{g} / \mathrm{mL}$ and $75 \mu \mathrm{g} / \mathrm{mL})$ and TC $(1 \mu \mathrm{g} / \mathrm{mL}$ and $1.5 \mu \mathrm{g} / \mathrm{mL}$ )

\section{Comparison of the results of PLS, PCR, and ILS methods} The accuracy of each method was compared with respect to the added standard. Their correlation matrix and $p$ values were calculated via the Pearson method. Results showed that there is no significant difference and PLS, PCR, and ILS method could be confidentially used for the simultaneous determination of ET and TC. All correlation matrix and $p$ values were monitored for $\mathrm{ET}$ and $\mathrm{TC}$ in Table 3.

\section{Application of the proposed multivariate calibration methods}

The developed and validated multivariate calibration PLS, PCR, and ILS methods were successfully performed to the simultaneous assay of ET and TC in commercial mixture products (Etotio ${ }^{\circ}$ tablets). It was shown that the concentrations of both ET and TC in UV absorption spectra (Fig. 4) of the commercial tablet were analyzed by the proposed spectrophotometric method (Table 4). The predictions of ten replicates were accomplished. High analytical recovery values confirm that there is no significant loss due to the extraction procedure and filtering of the pharmaceutical formulation. Results that were obtained for each active substance have good

Table 4 Analysis results of ET and TC in commercial mixture products (Etotio ${ }^{\circledR}$ tablets) using the multivariate calibration methods (PLS, PCR, and ILS)

\begin{tabular}{llllll}
\hline Commercial preparation & Method & Active substance & Mean \pm SD & Recovery \% & RSD \% \\
\hline Etotio $^{\oplus}$ tablets & PLS & ET (400 mg) & $396.5 \pm 14.21$ & 99.12 & 3.583 \\
& & TC (8 mg) & $7.919 \pm 0.284$ & 98.98 & 3.591 \\
& PCR & ET (400 mg) & $395.57 \pm 10.26$ & 99.39 & 2.582 \\
& TC (8 mg) & $7.94 \pm 0.326$ & 99.25 & 9.099 \\
& ILS & ET (400 mg) & $393.31 \pm 4.23$ & 98.33 & 9.08 \\
\hline
\end{tabular}


agreement with those declared by the manufacturing laboratories (Table 3).

\section{Conclusion}

The main goal of the proposed work is to develop and validate the novel chemometrics-assisted algorithm for the simultaneous determination of ET and TC in pharmaceuticals via chemometrics-assisted spectrophotometry method. According to the obtained data, three different chemometrics algorithm exhibited good accuracy and their correlation matrixes showed that there is no difference between each algorithm. Thus, each of them could be confidently used in the simultaneous determination of those pharmaceuticals. These proposed method presents a good alternative to chromatographic separations in routine quality control samples without using mobile phase or any other separation apparatus. Generally, chemometrics methods are very convenient techniques for the simultaneous analysis of multiple compounds in which the overlap of the spectra of the active compounds creates an interference that makes it impossible to determine the concentrations of each compound via classical linear regression equations. According to our results, PLS, PCR, and ILS statistically measure both ET and TC with respect to the $p$ values. Correlation matrix confirmed that each method has a very small difference and the prediction power of PLS and PCR is relatively better. Another advantage of the proposed method is that all analysis was performed neither derivatization nor ratio spectra modes which are expensive and time-consuming steps. Besides, the simplicity of the chemometric calibration methods comes from the ability to evaluate a huge amount of samples in a short time as accurately and precisely in comparison with chromatographic methods. The obtained results demonstrated that the proposed spectrophotometric method can be applicable as a possible alternative method for the simultaneous determination of ET and TC in the routine quality control analysis of pharmaceutical industries.

\section{Abbreviations}

ET: Etodolac; HPLC: High-performance liquid chromatography; HPTLC: Highperformance thin-layer chromatography; ILS: Inverse least squares; NSAID: Nonsteroidal anti-inflammatory drug; PCA: Principal component analysis; PCR: Principal component regression; PLS: Partial least squares; RE: Relative error; RSD \%: Percent relative standard deviation;

TC: Thiocolchicoside; UV: Ultraviolet

\section{Acknowledgements}

Not applicable.

\section{Funding}

This work has not been financially supported.

\section{Availability of data and materials}

Research data have been provided in the manuscript and supporting information.

\section{Authors' contributions}

This study was designed by FDM, MA, and OS, and EP performed the experimental work. The manuscript was written through the contributions of MA and FDM. The statistical analysis was performed by MA and OS. The journal format design and English editing were written through the contributions of MA. All authors have given approval to the final version of the manuscript

\section{Competing interests}

The authors declare that they have no competing interests.

\section{Publisher's Note}

Springer Nature remains neutral with regard to jurisdictional claims in published maps and institutional affiliations.

\section{Author details}

'Department of Medical Laboratory Techniques, Health Services Vocational Training School, Ataturk University, 25240 Erzurum, Turkey. ${ }^{2}$ Department of Analytical Chemistry, Faculty of Pharmacy, Ataturk University, 25240 Erzurum, Turkey.

Received: 9 January 2019 Accepted: 25 March 2019

Published online: 10 April 2019

\section{References}

Alagar R, Priyadarshini C, David B, Rao K, Selvakumar D. Validated RP-HPLC method for simultaneous estimation of etodolac \& thiocolchicoside in pharmaceutical tablet dosage form. J Pharm Res. 2012;5(8):4577-9.

Andelman S. Etodolac, aspirin, and placebo in patients with degenerative joint disease: a twelve-week study. Clin Ther. 1983:5(6):651-61.

Biziere K, Huguet F, Narcisse G, Breteau M. Affinity of thiocolchicoside and thiocolchicoside analogues for the postsynaptic GABA receptor site. Eur J Pharmacol. 1981;75(2-3):167-8.

Dhiware AD, Gandhi SV, Deshpande PB, Bhavnani VS. A simple and sensitive rphplc method for simultaneous estimation of etodolac and thiocolchicoside in combined tablet dosage form. Int J Pharm Pharm Sci. 2012;4(4):214-6.

Dinç E, Baleanu D. Spectrophotometric quantitative determination of cilazapril and hydrochlorothiazide in tablets by chemometric methods. J Pharm Biomed Anal. 2002;30(3):715-23.

European Pharmacopoeia 6th edition, European Directorate for the Quality of Medicines, Conseil de l'Europe, Secretariat General, France, 2007, ISBN 13: 9789287160546.

Hasegawa K, Ohashi Y, Ishikawa K, Yasue A, Kato R, Achiwa Y, et al. Expression of cyclooxygenase-2 in uterine endometrial cancer and anti-tumor effects of a selective COX-2 inhibitor. Int J Oncol. 2005:26(5):1419-28.

Lynch S, Brogden RN. Etodolac. Drugs. 1986;31(4):288-300.

Mascia MP, Bachis E, Obili N, Maciocco E, Cocco GA, Sechi GP, et al. Thiocolchicoside inhibits the activity of various subtypes of recombinant GABA A receptors expressed in Xenopus laevis oocytes. Eur J Pharmacol. 2007;558(1):37-42.

Matsuyama M, Yoshimura R, Hase T, Chargui J, Yoshimura N, Touraine JL. Administration of the selective cyclooxygenase (COX)-2 inhibitor etodolac prolongs cardiac allograft survival in a mouse model. Mol Med Rep. 2010; 3(5):771-4.

Pandey R, Patil PO, Bari SB, Dhumal DM. Simultaneous estimation of etodolac and thiocolchicoside in bulk and in tablet formulation by uvspectrophotometry. Chem Ind Chem Eng Q. 2014;20(1):9-17.

Patel A, Shah B. RP-HPLC method development and validation using factorial design for simultaneous estimation of thiocolchicoside and etodolac with forced degradation studies. J Pharm Sci Bio Res. 2014;4(6):374-82.

Perucca E, Poitou P, Pifferi G. Comparative pharmacokinetics and bioavailability of two oral formulations of thiocolchicoside, a GABAmimetic muscle relaxant drug, in normal volunteers. Eur J Drug Metab Pharmacokinet. 1995;20(4):301-5.

Rathod K, Patel J. Simultaneous estimation of etodolac and thiocolchicoside in their combined marketed formulation by RP-HPLC. Int J PharmTech Res. 2012:4(4):1513-9.

Riahi S, Hariri M, Ganjali MR, Norouzi P. Rapid and direct spectrofluorometric and chemometrics methods for the simultaneous determination of two dansyl derivatives. Spectrosc Lett. 2010;43(3):226-34. 
Riendeau D, Percival M, Boyce S, Brideau C, Charleson S, Cromlish W, et al. Biochemical and pharmacological profile of a tetrasubstituted furanone as a highly selective COX-2 inhibitor. Br J Pharmacol. 1997;121(1):105-17.

Sankar A, Vetrichelvan T, Venkappaya D. Simultaneous estimation of ramipril, acetylsalicylic acid and atorvastatin calcium by chemometrics assisted UVspectrophotometric method in capsules. Acta Pharma. 2011;61(3):283-96.

Sechi G, De Riu P, Mameli O, Deiana GA, Cocco GA, Rosati G. Focal and secondarily generalised convulsive status epilepticus induced by thiocolchicoside in the rat. Seizure. 2003;12(7):508-15.

Sorouraddin MH, Khani MY, Amini K, Naseri A, Asgari D, Rashidi MR. Simultaneous determination of 6-mercaptopurine and its oxidative metabolites in synthetic solutions and human plasma using spectrophotometric multivariate calibration methods. Biolmpacts: Bl. 2011;1(1):53-62.

Syamala S. Development and validation of new RP-HPLC method for simultaneous estimation of drug thiocolchicoside and etodolac in tablet dosage form. Indian J Pharm Biotech. 2016;4(4):180.

Tekade SW, Havele SS, Dhaneshwar SR. Validated HPTLC method for simultaneous estimation of etodolac and thiocolchicoside in bulk drug and formulation. J Pharm Biomed Sci. 2012;23(07):1-4.

Thankappan S, Parmar A, Sailor B, Vekariya K, Shah D. Simultaneous estimation of etodolac and thiocolchicoside by uv spectrophotometric method in tablet formulation. Int J Pharm Innov. 2012:2(2):192-200,

Tiwari R, Pillai S. Spectrophotometric estimation of etodolac and thiocolchicoside in tablet dosage. Int J Pharm Technol. 2011:4(12):1891-5.

\section{Submit your manuscript to a SpringerOpen ${ }^{\circ}$ journal and benefit from:}

- Convenient online submission

- Rigorous peer review

- Open access: articles freely available online

- High visibility within the field

- Retaining the copyright to your article

Submit your next manuscript at $\boldsymbol{\nabla}$ springeropen.com 